\title{
Dynamics of Nonlinear Interactions among Forces with Lagged Effect
}

\author{
Jair Silvério dos Santos, Katia A. G. Azevedo \\ Departamento de Computação e Matemática, FFCLRP-Universidade de São Paulo, São Paulo, Brazil \\ Email: jair@ffclrp.usp.br, kandreia@ffclrp.usp.br
}

How to cite this paper: dos Santos, J.S. and Azevedo, K.A.G. (2017) Dynamics of Nonlinear Interactions among Forces with Lagged Effect. Applied Mathematics, 8, 1437-1444.

https://doi.org/10.4236/am.2017.810104

Received: April 25, 2017

Accepted: October 21, 2017

Published: October 25, 2017

Copyright @ 2017 by authors and Scientific Research Publishing Inc. This work is licensed under the Creative Commons Attribution International License (CC BY 4.0).

http://creativecommons.org/licenses/by/4.0/

\begin{abstract}
This paper is concerned with the dynamics of the steady state of a two-delayed nonlinear system of functional differential equations. The stability of the steady state together with its dependence on the magnitude of time delays has been examined by means of characteristic equation corresponding to the nonlinear equation. General criteria for stability involving the two-delay equations have been obtained.
\end{abstract}

\section{Keywords}

Hopf Bifurcation, Business Cycle, Stability, Two-Delayed Kaldor-Kalecki Model, Fluctuations Phenomenum

\section{Introduction}

Differential equations models that incorporate the history of the phenomenum into the model have been extensively considered as model for many problems because of a commum feature to them, which is, the appearance of oscillations, a fact which is very important in the model problems coming from ecology and mathematical economics [1] [2] [3] [4] [5]. Since delay-differential equations share many properties with ordinary differential equations, we have use methods and techniques of geometric dynamical systems theory that have been implemented in functional differential equations to describe the dynamics of flow associated with the system of equations

$$
\left\{\begin{array}{l}
\dot{u}(t)=f(u(t), v(t)) \\
\dot{v}(t)=h(u(t), v(t))+g(u(t-r), v(t-\sigma))
\end{array}\right.
$$

where $f, h, g: R^{2} \rightarrow R$ are sufficiently smooth functions, $r$ and $\sigma$ are non negative constants. Assume that $f, h$ and $g$ vanish at $P_{0}=\left(u_{0}, v_{0}\right), f_{u}\left(P_{0}\right)=\kappa_{11}$, 
$f_{v}\left(P_{0}\right)=\kappa_{12}, h_{u}\left(P_{0}\right)=\kappa_{21}, \quad h_{v}\left(P_{0}\right)=\kappa_{22}, g_{u}\left(P_{0}\right)=\delta$ and $g_{v}\left(P_{0}\right)=\kappa$, near an equilibrium point (see [2] [4] [6] [7]). The model represented by system (1) has been considered because it appears as generator of self-sustaining cycles with time lags incorporated in the nonlinearities. Recently, dynamical systems with delays have been found in biology and economics, and delay dependence on the state of the system cannot be eliminated by any change of variables (see [2] [8] [9] [10] references cited there).

When $\sigma=r$, the authors in [11] studied the stability and Hopf bifurcation of the ratio-dependent predator-prey system similar to (1). Yuan in [12] exploited the behaviuor of plankton-population models, one with instantaneous predation, another with delayed predation and has obtained conditions to guarantee the coexistence of two species, and addresses the stability and bifurcation under different density of fish, with or without maturation time delay. We have decided to keep to the model represented by system 1 with $\sigma \neq r$ to improve understanding of the combined effects of functional response and time delays on the dynamics of predator-prey systems. Point $P_{0}$ that is equilibrium point for the system 1, therefore also equilibrium of (2). Taking a delay as a paramenter $(\sigma>0)$, our purpose is to relate the dynamics of the two systems in the neighbourhood of $P_{0}$.

The linear problem around $P_{0}$, associated to System (1), is given by

$$
\left\{\begin{array}{l}
\dot{u}(t)=\kappa_{11} u(t)+\kappa_{12} v(t) \\
\dot{v}(t)=\kappa_{21} u(t)+\kappa_{22} v(t)+\delta u(t-r)+\kappa v(t-\sigma)
\end{array}\right.
$$

and its characteristic equation is given by

$$
p(\lambda)=\left(\kappa_{11}-\lambda\right)\left[\kappa_{22}-\lambda+\kappa \mathrm{e}^{-\lambda \sigma}\right]-\kappa_{12}\left(\kappa_{21}+\delta \mathrm{e}^{-\lambda r}\right)=0 .
$$

By assuming that

$$
\kappa_{11}=0, \kappa_{12}>0, \kappa_{22}<0, \kappa_{21}<-\delta<0 \text { and } \kappa>0,
$$

the Equation (3) becomes

$$
p(\lambda)=\lambda^{2}-d \lambda-b \lambda \mathrm{e}^{-\lambda \sigma}-a \mathrm{e}^{-\lambda r}+c=0,
$$

where $a=\delta \kappa_{12}, b=\kappa, c=-\kappa_{12} \kappa_{21}$ and $d=\kappa_{22}$ and $a, b, c$ and $-d>0$. If $\sigma=r$, we can choose $a, b, c, d$ for the Equation (5) as the characteristic Equation (3.5) examined [2].

For a moment assume that either $d=0$ and $c<a$ or $a>0$ and $c<0$, so it is not true that all roots of the Equation (5) have negative real part neither, since on the real axis $p(0)=c-a<0$ and $p(\lambda) \rightarrow \infty$ as $\lambda \rightarrow \infty$, then system 1 will be unbounded solutions.

Because of the two delays, the analysis on how the roots of (5) locate with respect to the imaginary axis is a classical problem that, besides being important in itself, plays an important role in the study of asymptotic behavior in the theory of delayed differential equations (see [6]). In view of the difficulty to find all the values of the parameters for which all the roots $\lambda$ in (5) have negative real parts, only the special situations of (5) are considered [1] [2] [6] [11] [13]. To 
analyze the behavior dynamics of System (1) close to equilibrium solution it is necessary to have detailed information about the behavior of the eigenvalue of the linear equation associated to it, and so this problem can be reduced to the fact that all roots of Equation (5) have negative real part. There have been many attempts to describe the region $K$ in the parameters space in which the solutions of (5) approach equilibrium solution (see [7] section 11.2). In fact, each point that belongs to the boundary of $K, \partial(K)$, is of primary interest because it represents the point where the equilibrium point of System (1) can undergo a bifurcation from stability to instability. In the analysis, we have described the region $K$ for System (1) in Theorem 1 and found parameters belonging to $\partial(K)$, which correspond to the point where the equilibrium of System (1) switches from being stable to unstable with periodic oscillations as the parameter

$\sigma$ crosses the value $\frac{\pi}{2}$.

\section{Stability}

Let $\lambda=x+i y$ be a solution of Equation (5). Separating real and imaginary part in (5) one obtains the following equations system for $x$ and $y$.

$$
\left\{\begin{array}{l}
x^{2}-\left(y^{2}-c\right)-d x-b \mathrm{e}^{-\sigma x}[x \cos y \sigma+y \sin y \sigma]-a \mathrm{e}^{-r x} \cos y r=0 \\
2 x y-d y+b \mathrm{e}^{-\sigma x}[x \sin y \sigma-y \cos y \sigma]+a \mathrm{e}^{-r x} \sin y r=0
\end{array}\right.
$$

In order to simplify notation we set

$$
\bar{x}=\frac{b+\sqrt{b^{2}+2 a}}{2}, \bar{y}=\frac{\bar{x}}{2}+\frac{b}{2}-\frac{d}{2}+\sqrt{\left(\frac{\bar{x}}{2}+\frac{b}{2}-\frac{d}{2}\right)^{2}+a+c .}
$$

Theorem 1. Assume that (4) is satisfied and

$$
\begin{gathered}
d<\min \{-\sqrt{2} b / 2,-\sqrt{2 c}\}, 2 r<2 \sigma<\pi, \\
4 a+(b+d)^{2}<4 c, \quad \bar{y}<2, \\
b<2 \sqrt{2 \Upsilon(a, c)\left(d^{2}-2 c\right)}, \text { where }\left(8 c^{2}+a^{2}\right) \Upsilon(a, c)=c^{2}-a^{2},
\end{gathered}
$$

Then, there is $\epsilon_{0}>0$ so that if $b<2 \epsilon_{0}$, all roots of Equation (5) have negative real part.

Proof: Observe that $a, b$ and $c$ are positive, $d$ is negative and $c>a$ (see (4)). Suppose that $y=0$ in (6). The system has been reduced to $x^{2}-\left(b \mathrm{e}^{-\sigma x}+d\right) x-a \mathrm{e}^{-r x}+c=0$. If $\vartheta(x)=x^{2}-\left(b \mathrm{e}^{-\sigma x}+d\right) x-a \mathrm{e}^{-r x}+c$ then $\vartheta(0)>0$. If $x>0$, the first inequality in (7b) results in $\vartheta(x)>$ $x^{2}-(b+d) x-a+c>0$. From this it follows that $p(\lambda)=0$ and so has no positive solution with null imaginary part. The roots of the system (6) with null real part must be roots of the system

$$
\left\{\begin{array}{l}
\text { by } \sin y \sigma+a \cos y r=-\left(y^{2}-c\right) \\
-b y \cos y \sigma+a \sin y r=d y
\end{array}\right.
$$

We will show that system (6) has no purely imaginary solution. Suppose 
$y \neq 0$ is a solution of (8), then one must have

$$
\sin ((\sigma-r) y)=\frac{\left(c-y^{2}\right)^{2}+\left(d^{2}-b^{2}\right) y^{2}-a^{2}}{2 a b y}:=\rho(y) .
$$

It is clear that $\rho(y)=-\rho(-y)$, thus we will consider only $y>0$. A direct calculation shows that $2 b a y^{2} \dot{\rho}(y)=3 y^{4}+\left(d^{2}-b^{2}-2 c\right) y^{2}-\left(c^{2}-a^{2}\right)$ and that

$$
y_{0} \sqrt{6}=\left[\sqrt{\left(d^{2}-\left(2 c+b^{2}\right)\right)^{2}+12\left(c^{2}-a^{2}\right)}-\left(d^{2}-\left(2 c+b^{2}\right)\right)\right]^{\frac{1}{2}} \text {, }
$$

is a minimum global point of $\rho$ on the interval $(0, \infty)$.

Since $a b y^{2} \dot{\rho}(y)=a b y \rho(y)+y^{4}-\left(c^{2}-a^{2}\right) \quad$ and $\dot{\rho}\left(y_{0}\right)=0$, then 3aby $_{0} \rho\left(y_{0}\right)=2\left(c^{2}-a^{2}\right)+\left(d^{2}-2 c-b^{2}\right) y_{0}^{2}$. From the first inequality in $(7 \mathrm{a})$ and the inequality of (7c) we notice that $\rho\left(y_{0}\right)>1$ and (5) have no purely imaginary root.

Let consider the sets $S_{i}$ for $i \in\{0,1,2, \cdots, 6\}$ given by

$$
\begin{aligned}
& S_{0}=\left\{(x, y) \in \mathbb{R}^{2}: 0<x \leq \bar{x} \text { and } 0<\bar{y} \leq y\right\} ; \\
& S_{1}=\left\{(x, y) \in \mathbb{R}^{2}: 0<4 \sigma y \leq \pi \text { and } 2 x>b\right\} ; \\
& S_{2}=\left\{(x, y) \in \mathbb{R}^{2}: \pi \leq 4 \sigma y \leq 2 \pi \text { and } 0<y \leq x\right\} ; \\
& S_{3}=\left\{(x, y) \in \mathbb{R}^{2}: \pi \leq 2 \sigma y \leq 2 \pi \text { and } x>0\right\} ; \\
& S_{4}=\left\{(x, y) \in \mathbb{R}^{2}: 0<y \leq x \text { and } y \geq \bar{x}\right\} ; \\
& S_{5}=\left\{(x, y) \in \mathbb{R}^{2}: 0<x \leq y \text { and } x \geq \bar{x}\right\} ; \\
& S_{6}=\left\{(x, y) \in \mathbb{R}^{2}: \pi \leq 4 \sigma y \leq 2 \pi \text { and } 0<x \leq y\right\} .
\end{aligned}
$$

For each $(x, y) \in R_{+}^{2}$, the first equation in (6) becomes equivalent to

$$
\Gamma_{1}(x, y)=: x-y-\frac{d x-c}{x+y}-b \mathrm{e}^{-x \sigma} \zeta_{1}(x, y)-\frac{\mathrm{e}^{-x r}}{x+y} a \cos y r=0,
$$

where $\zeta_{1}(x, y)=(x+y)^{-1}[x \cos y \sigma+y \sin y \sigma]$. We notice that

$$
\left|\zeta_{1}(x, y)\right| \leq 1 \text { and } \frac{-a \mathrm{e}^{-r x}}{x+y} \leq \frac{-a \mathrm{e}^{-r x} \cos r y}{x+y} \leq \frac{a \mathrm{e}^{-r x}}{x+y} \leq \frac{a \mathrm{e}^{-r x}}{y} .
$$

If $\epsilon^{*}(x, y)=-y^{2}+(b+x-d) y+a+c$, then it follows from (13) that $y \Gamma_{1}(x, y)<\epsilon^{*}(x, y)$. If $(x, y) \in S_{0}$, we can verify that $\max \left\{\epsilon^{*}(x, y):(x, y) \in S_{0}\right\}<0$ and so, Equation (12) has no solution belonging to $S_{0}$. In fact, function $2 y(x)=(b+x-d)+\sqrt{(b+x-d)^{2}+4(a+c)}$ is increasing in $x$ and yeilds the positive solution for the second-degree equation at $y$ given by $\epsilon^{*}(x, y)=0$.

The second equation in (6) is equivalent to

$$
\Gamma_{2}(x, y)=2 x y-d y+b \mathrm{e}^{-\sigma x}[x \sin \sigma y-y \cos \sigma y]+a \mathrm{e}^{-r x} \sin r y=0 .
$$

Since $\Gamma_{2}(x, y)>y(2 x-b)>0$ for $(x, y) \in S_{1}$, there is no solution of the Equation (14) that belongs to $S_{1}$. It is clear that $x \sin \sigma y-y \cos \sigma y \geq 0$ for $(x, y) \in S_{2}$. Moreover, for all $(x, y) \in S_{2}$, we have 
$\Gamma_{2}(x, y) \geq 2 x y-d y+a \mathrm{e}^{-r x} \sin r y>0$. Thus, there is no solution of (12) that belongs to $S_{2}$. Let consider $(x, y) \in S_{3}$, then $\Gamma(x, y) \geq 2 x y-d y$ is positive because $d<0$ (see (7a)). Hence, there is no solution of (12) that belongs to $S_{3}$. For each $(x, y) \in S_{4}$, we define $\zeta_{2}(x, y)=(x y)^{-1}[x \sin y \sigma-y \cos y \sigma]$. We can check directly that $y \zeta_{2}(x, y) \geq-2$ and $y \sin y r \geq-x$. Then, $\Gamma_{2}(x, y)>\left(x^{-1} y^{-3}\right)\left[2 y^{2}-b y-a\right]$ is positive if $y \geq \bar{x}$ (see (7)), hence (14) has no solution at $S_{4}$. Let $(x, y) \in S_{5}$. Because, $x \zeta_{2}(x, y) \geq-2$ and

$(x y)^{-1} \sin (y r)>-x^{-2}$, then $x^{2} \Gamma_{2}(x, y)>x^{-1} y^{-1}\left(2 x^{2}-2 b x-a\right)$ is positive if $x>\bar{x}$ (see (7)) and so, has no solution at $S_{5}$. Assume $(x, y) \in S_{6}$, since $x y \geq x^{2}, \quad-b y \sqrt{2} / 2<-b y e^{-\sigma x} \cos \sigma y, \quad \Gamma_{2}(x, y)>(-d y-b y \sqrt{2} / 2)$ is positive if $d<-b \sqrt{2} / 2$ (see (7a)). Hence, there is no solution for (12) that belongs to $S_{6}$.

By general arguments on the compactness of the interval $\left[0, \frac{\pi}{4}\right]$ and the continuity of $\rho$, we arrive at the existence of a $\epsilon_{0}>0$ so that System (6) has no solution belonging to $S_{\epsilon_{0}}=\left\{(x, y) \in R^{2}: 0 \leq x \leq \epsilon_{0}, 0 \leq \sigma y \leq \frac{\pi}{4}\right\}$. If $R_{+}^{2}=\left\{(x, y) \in R^{2}, x>0, y>0\right\}$, conditions (7a), (7b), (7c) and $b<2 \epsilon_{0}$ imply that $R_{+}^{2} \subset \mathcal{S} \cup S_{\epsilon_{0}}$, where $\mathcal{S}=\bigcup_{i=0}^{6} S_{i}$ (see (11)). So, it can be proven that System (6) has no solution that lies at $\mathrm{Cl}\left(R_{+}^{2}\right)$ and so the proof of the Theorem is complete.

Corollary 1. Let $a, b, c, d$ be given by (5), satisfing $d<\min \{-\sqrt{2} b / 2,-\sqrt{2 c}\}$, $(7 b)$ and (7c). Then, there is $\epsilon_{0}>0$ so that if $\bar{y}<\epsilon_{0}$ the equilibrium solution of System (1) is asymptotically stable for all $\sigma$ and $r$ (see (7)).

Proof: Let consider $\epsilon_{0}$ and $S_{\epsilon_{0}}$ given in Theorem 1. It is easy to see that if $\bar{y}<\epsilon_{0}$ then $S_{i} \subset S_{\epsilon_{0}}$ for $i \in\{1,2,3,4\}$ and in this case $\mathrm{Cl}\left(R_{+}^{2}\right) \subset S_{\epsilon_{0}} \cup S_{0} \cup$ $S_{5} \cup S_{6}$ is indepentent from $r$ and $\sigma$ (see (11)).

\section{Hopf Bifurcation}

A search for purely imaginary solutions of Equation (6) plays a key role in the analysis of stability and bifurcation of periodic solution of System (1). We will find solutions for Equation (6) with real part null, which actully solutions of the System (8).

Theorem 2. Assume (4), $d=-\sqrt{2} b / 2, \sigma=\pi / 2$. Given $a, b>0$, consider $r^{*}$ so that $r^{*}<\frac{b}{a}\left(1-\frac{\sqrt{2}}{2}\right)<\frac{\pi}{2}$. For $a, b$ and $r^{*}$ there exists $0<y^{*}<1 / 2$, solution of the second equation of System (8). If $c=b y^{*} \sin \left(\pi y^{*} / 2\right)+$ $a \cos \left(r^{*} y^{*}\right)+\left(y^{*}\right)^{2}$, then $\left(\pi / 2, r^{*}, y^{*}\right)$ is the solution of System 8. Moreover, $\mathfrak{R}\left(\left.\dot{\lambda}\left(\frac{\pi}{2}\right)\right|_{x=0}\right)>0$.

Proof: Consider $d=-\sqrt{2} b / 2$ in the second equation of the System (8). We 
set $H(y)=a \sin (r y)$ and $G(y)=y b\left(-\frac{\sqrt{2}}{2}+\cos (\pi y / 2)\right)$. It is easy to see that $G(0)=G(1 / 2)=0$ and $G$ is concave with a maximum at $\bar{y}, \bar{y}$ is near $y=0.285281$. Clearly, $H(y)$ is an increasing function for $0<y<1 / 2$ and $H(0)=0$. If $H^{\prime}(0)=a r<b\left(1-\frac{\sqrt{2}}{2}\right)=G^{\prime}(0)$ with $r<\pi / 2$, there exists $y$, so that $H(y)=G(y)$. Thus, given $a, b$ so that $\frac{b}{a}\left(1-\frac{\sqrt{2}}{2}\right)<\frac{\pi}{2}$, consider $r^{*}<\frac{b}{a}\left(1-\frac{\sqrt{2}}{2}\right)$ and for this $r^{*}$ consider $y^{*}$ given by $H(y)=G(y)$. It is possible to find $c$ so that $y^{*}$ is the solution of $b y \sin (\pi y / 2)+a \cos \left(r^{*} y\right)$ $+y^{2}=c$, which is the first equation of System 8 or else consider by $\sin \left(\pi y^{*} / 2\right)+a \cos \left(r^{*} y^{*}\right)+\left(y^{*}\right)^{2}=c$. Therefore $\left(\pi / 2, r^{*}, y^{*}\right)$ is the solution of System 8 for $a, b$ and $c$ given above.

Now, by using implicit derivative in (5) we can show that

$$
\begin{gathered}
\mathfrak{R}\left(\left.\dot{\lambda}(\sigma)\right|_{x=0}\right)=b y^{2}[2 y \sin \sigma y-d \cos \sigma y]+\text { bar } \cos (\sigma-r) y \\
\mathfrak{R}\left(\left.\dot{\lambda}(\pi / 2)\right|_{x=0}\right)=b\left(y^{*}\right)^{2}\left[2 y^{*} \sin \pi / 2 y^{*}-d \cos \pi / 2 y^{*}\right]+b \operatorname{bar} \cos \left(\pi / 2-r^{*}\right) y^{*}>0
\end{gathered}
$$
is positive because $0<y^{*}<1 / 2$ and $a, b,-d>0$.

A simple observation yields the following important corollary.

Corollary 2. Let a,b,c,d satisfy the hypotheses of Theorem 2. Then for each $(k, m) \in Z \times Z$ there corresponds $\left(\sigma_{k}, r_{m}\right)$ given by, $y^{*} \sigma_{k}=y^{*} \pi / 2+2 k \pi$ and $y^{*} r_{m}=y^{*} r^{*}+2 m \pi$ so that $\left(\sigma_{k}, r_{m}, y^{*}\right)$ solves System 8 . Moreover, $\mathfrak{R}\left(\left.\dot{\lambda}\left(\sigma_{k}\right)\right|_{x=0}\right)>0$ and the equilibrium solution of System (1) undergoes a Hopf bifurcation at the points $\sigma_{k}, k=0,1,2, \cdots$.

\section{Two-Delayed Kaldor-Kalecki Model}

Consider a Kaldor-Kalecki model of business cycle with two delays where memory-dependence may be not only due to regulation, but also to the inertia of institutional, technical systems including the deployment of research and development results described by

$$
\left\{\begin{array}{l}
\dot{Y}(t)=\alpha[I(Y(t), K(t))-S(Y(t), K(t))] \\
\dot{K}(t)=\beta I(\tilde{m} Y(t)+(1-\tilde{m}) Y(t-\eta), m K(t-\tau)+(1-m) K(t))-q K^{b_{1}-d_{0}}(t)
\end{array}\right.
$$

where $0<d_{0}<b_{1}, \tilde{m} \geq 0,0 \leq m \leq 1, Y$ is the gross product, $K$ the capital stock, $\alpha>0$ the adjustment coefficient in the goods market, $q \in(0,1)$ the depreciation rate of capital stock, $I(Y, K)$ and $S(Y, K)$ investment and saving are nonlinear and we assume are smooth enough fuctions, and $\eta, \tau>0$ are time lags representing delays for the investment in the capital stock due to past investment decision, which we assume are in both delays the gross product and the human or physical capital stockrespectively. Let $a_{0}, b_{0}$ and $c_{0}$ be real numbers such as $0<a_{0}<c_{0}, b_{0} \neq b_{1}, a_{0}+b_{0} \neq c_{0}+d_{0}$. If 


$$
\begin{aligned}
& \gamma^{*}=a_{0} d_{0}-b_{0} c_{0}-\left(b_{1}-d_{0}\right)\left(a_{0}-c_{0}\right)^{-1}, \text { we define } \\
& I(Y, K)=Y^{a_{0}}(t) K^{b_{0}}(t)-q_{1} K^{\left(b_{0}-d_{0}\right)} \\
& \text { and } S(Y, K)=Y^{c_{0}}(t) K^{d_{0}}(t)-q_{1} Y^{\left(c_{0}-a_{0}\right)} .
\end{aligned}
$$

Then, $P_{0}=\left(Y_{0}, K_{0}\right)=\left(\left(1+q \beta^{-1}\right)^{\frac{1}{b_{0}-b_{1}}} ;\left(1+q \beta^{-1}\right)^{\frac{d_{0}}{b_{1}-b_{0}}}\right)$ is a solution of the non-linear system

$$
\left\{\begin{array}{l}
I(Y(t), K(t))-S(Y(t), K(t))=0, \\
I(Y(t), K(t))-q \beta^{-1} K^{\left(b_{1}-d_{0}\right)}(t)=0 \\
I_{Y}(Y(t), K(t))-S_{Y}(Y(t), K(t))=0 .
\end{array}\right.
$$

which is also a steady state for the system 16 . The characteristic equation of the linear problem around $P_{0}$, associated to System 16, is given by the Equation (5) where

$$
\begin{aligned}
& a=\left(I_{K}\left(P_{0}\right)-S_{K}\left(P_{0}\right)\right)(1-\tilde{m}) I_{Y}\left(P_{0}\right), b=m I_{K}\left(P_{0}\right), \\
& c=\left(I_{K}\left(P_{0}\right)-S_{K}\left(P_{0}\right)\right) \tilde{m} I_{Y}\left(P_{0}\right) \text { and } d=(1-m) I_{K}\left(P_{0}\right)-q .
\end{aligned}
$$

If we assume $0<(1-m) I_{K}\left(P_{0}\right)<q, \quad I_{K}\left(P_{0}\right)-S_{K}\left(P_{0}\right)<0, \quad I_{Y}\left(P_{0}\right)>0$, and $I_{K}\left(P_{0}\right)>0$, then $a, b$, and $c$ are positive real numbers and $d<0$. If $a, b, c, d, r$ and $\sigma$ satisfy all conditions of theorem 1 the steady state $P_{0}$ of the KaldorKalecki system 16 is asymptocaly stable. If the conditions of corollary 1 are satisfied the stability is delay-independent. If the conditions of theorem 2 are satisfied then the steady state $P_{0}$ becomes unstable and undergoes a Hopf bifurcation. This Hopf bifurcation is the first one of a cascade taking place as the delays go to infinity. The structure of this cascade is described by corollary 2 .

\section{Concluding Remarks}

This paper studies dynamic characteristics of feed-back effects that incorporate the memory of the phenomenum into the model because of a commum feature to them, which is, the appearance of oscillations, a fact which is very important in the model problems coming from mathematical economics or ecology (see 1). We have chosen an approach that makes it clear how the delay phenomena and anti-damping force dominate, lead either asymptotic stability or fluctuations to system 1, and it can also make trajectories converge to the other limit cycle which may be stable or not. We apply our results to describe Kaldor-Kaleck model dynamics 16, which can be used to model decision-making dynamics in business cycle. Ours results describe special circumstances which show that equilibrium point of system 1 can either have a stable fixed point surrounded by an unstable cycle that appears because Hopf bifurcation.

\section{References}

[1] Huang, W. (2013) Information Lag and Dynamic Stability. Journal of Mathematical Economics, 44, 513-529. 
[2] Pal, P.J. and Mandal, P.K. (2014) Bifurcation Analysis of a Madified Leslie-Gower Predator-Prey Model with Beddington-DeAngelis Functional Response and Strog Allee Effect. Mathematics and Computers in Simulation, 97, 123-146. https://doi.org/10.1016/j.matcom.2013.08.007

[3] Rustichini, A. (1989) Hopf Bifurcation for Functional Differential Equations of Mixed. Journal of Dynamics and Differential Equations, 1, 145-177. https://doi.org/10.1007/BF01047829

[4] Santos, J.S. and Dos Reis, J.G. (1996) Unbounded Continum of Periodic Solutions for a Autonmous Delay Equation. Journal of Mathematical Analysis and Applications, 203, 840-849. https://doi.org/10.1006/jmaa.1996.0415

[5] Wu, X.P. (2012) Zero-Hopf Bifurcation Analysis of a Kaldor-Kalecki Model of Business Cycle with Delay. Nonlinear Analysis, Real World Appiled, 13, 736-754. https://doi.org/10.1016/j.nonrwa.2011.08.013

[6] Aki, S.M.T. and Godoy, S.M.S. (1981) Permanence of Stability for a Class of System of Differential Equations with Two Delays. Nonliner Analysis, 1, 172-184.

[7] Hale, J.K. and Lunel, S.M.V. (1993) Introduction to Functional Differential Equations. Springer Verlag-New York. https://doi.org/10.1007/978-1-4612-4342-7

[8] Matsunaga, H. (2008) Delay-Dependent and Delay-Independent Stability Criteria for a Delay Differential System. Proceedings of the American Mathematical Society, 136, 4305-4312. https://doi.org/10.1090/S0002-9939-08-09396-9

[9] Ruan, S. and Wei, J. (1999) Periodic Solutions of Planar Systems with Two Delays. Physica D, 129-A, 1017-1032.

[10] van den Driessche, P. and Wu, J. (1981) Stabilization Role of Inhibitory Self-Connections in a Delayed Neuralnetwork. Physica D, 150, 84-90.

[11] Liu, Z. and Yuan, R. (2004) Stability and Bifurcation in a Delayed Predator-Prey System with Beddington-Deangelis Functional Response. Journal of Mathematical Analysis and Applications, 296, 521-527. https://doi.org/10.1016/j.jmaa.2004.04.051

[12] Yuan, Y. (2012) A Coupled Plankton System with Instantaneous and Delayed Predation. Journal of Biological Dynamics, 6, 148-165. https://doi.org/10.1080/17513758.2010.544409

[13] Faria, T. (2000) On a Planar System Modelling a Neuron Network with Memory. Journal of Differential Equations, 168, 129-49. https://doi.org/10.1006/jdeq.2000.3881 